\title{
Regional climate pattern during two millennia estimated from annual tree rings of Yaku cedar trees: a hint for solar variability?
}

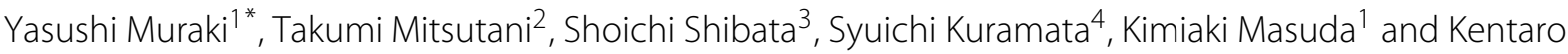 \\ Nagaya $^{1}$
}

\begin{abstract}
We analyzed trees that have survived on Yaku island (Yakushima) for 2,000 years. Quite surprisingly, the Fourier and wavelet analyses of the annual growth rate identified 2 cycles of periodicities of 11 and $(24 \pm 4)$ years during the Oort, Wolf, Spörer, Maunder, and Dalton minima. The 11-year periodicity originated from solar activity, while the (24 \pm 4)-year periodicity may be related to the Pacific Decadal Oscillation (PDO). In particular, we have discovered an 11-year periodicity in the meteorological daylight-hour data from Yakushima in the month of June during 1938 to 2013 and a 24-year periodicity in July. The growth rate of the tree rings may be affected by the variation of the daylight hour.
\end{abstract}

Keywords: Sun-earth relation; Tree rings; Grand minima; Dendrochronology; Climate change

\section{Findings}

\subsection{Introduction}

\subsubsection{Environment of Yakushima}

Yakushima (Yaku island) is located south of Kyushu in Japan (its geographical location is $30^{\circ} 20^{\prime} \mathrm{N}, 133^{\circ} 30^{\prime} \mathrm{E}$ ). Yakushima has been declared a world natural heritage site by UNESCO. On the island, there are mountains of 2,000 $\mathrm{m}$ height and cedar trees that have lived for more than 2,000 years. Cedar trees with a lifetime of more than 1,000 years are called Yakusugi (Yaku cedars). The island is also well known to experience heavy rainfall, with the annual rainfall reaching $4,500 \mathrm{~mm}$. We analyzed the annual tree rings of the Yakusugi and obtained very interesting results that we report in this paper.

On the southern shore of Yakushima runs the Kuroshio Current (a warm ocean current). The width of the current is about $100 \mathrm{~km}$, and it has high salinity (34.8 per mill) but is poor in plankton compared to the Oyashio Current (a cold current). The average temperature of the island is $23^{\circ} \mathrm{C}$ in the summer time and $16^{\circ} \mathrm{C}$ in winter. In July and August, the average high temperature is $30^{\circ} \mathrm{C}$, and

\footnotetext{
*Correspondence: muraki@stelab.nagoya-u.ac.jp

1 Solar-Terrestrial Environment Laboratory, Nagoya University, 464-8601

Nagoya, Japan

Full list of author information is available at the end of the article
}

the average low temperature is $24^{\circ} \mathrm{C}$. However, in June, the rainy season begins, and the sky over the island is covered with clouds. The total amount of precipitation reaches 800 $\mathrm{mm}$ in June.

\subsubsection{Research goals}

Here, we introduce two preceding studies based on tree rings. One was conducted by our group (Muraki et al. 2011) and the other by Brazilian scientists (Rigozo et al. 2005). In a previous work, we analyzed the tree rings of a 391-year-old cedar in Muroji. One of the main purposes of our research was to analyze the variation of the radiocarbon $\mathrm{C}^{14}$ during the Maunder minimum, and the results have been published (Miyahara et al. 2004).

However, an analysis was later performed on the same tree rings using a dendrochronology technique, which yielded an interesting result. During the Maunder minimum, the tree grew with a periodicity of 12 years. The 12-year periodicity may be connected to solar activity. However, in the analysis performed by Rigozo et al. (2005), evidence of solar activity was sparsely recognized in tree rings found in Chile. Instead, they detected the El Niño Southern Oscillation.

Lean and Rind (2008) analyzed 117 years of global meteorological data and found the effect of solar activity on the

\section{是 Springer}

(c) 2015 Muraki et al:- licensee Springer. This is an Open Access article distributed under the terms of the Creative Commons Attribution License (http://creativecommons.org/licenses/by/4.0), which permits unrestricted use, distribution, and reproduction in any medium, provided the original work is properly credited. 
climate at the latitudes of $30^{\circ}$ to $60^{\circ} \mathrm{N}$ and $30^{\circ}$ to $50^{\circ} \mathrm{S}$, with a peak amplitude of $0.5 \mathrm{~K}$.

In the southern hemisphere, the effect was identified over the South Atlantic Ocean. Therefore, we need to analyze another sample of trees collected from the area where the effect of solar variation was reported by Lean and Rind (2008).

In addition, a search is required to see if the same effect found by our group (Muraki et al. 2011) occurred during the Wolf and the Spörer minima. According to Lean and Rind (2008), in the northern hemisphere, the solar effect was recognized from the Ural Mountains to the Far East. Therefore, we analyzed the Yaku cedars that have survived over 1,000 years in the Far East, searching for evidence of solar activity hidden in the old tree rings. This paper reports the results of the analysis.

\subsection{Variation of annual tree rings}

The annual growth rate of the Yakusugi was measured by one of the authors (Takumi Mitsutani) using a special device with a microscope. The dates of each tree are listed in Table 1. The variation of the growth rate between 305 $\mathrm{CE}$ and $1000 \mathrm{CE}$ was obtained using two samples, while the data between $1254 \mathrm{CE}$ and $1935 \mathrm{CE}$ was prepared using six samples. Thus, the record of the variation during 1254 and 1935 is very reliable. The data were also compared with the growth rate of the cedar trees on Shikoku Island, located $500 \mathrm{~km}$ away from Yakushima, and a good correlation between them was confirmed. Therefore, we can use the data to represent the typical variations of the tree rings of the Pacific coast of Japan.

We estimated the typical variation of the tree rings in the following way: the annual growth rate of a tree ring was measured, i.e., two lines were drawn from the center of a tree to the edges, and the annual growth rate was measured along the line. Then, these data were averaged, and the value was regarded as the variation of the tree ring. For example, when we estimated the variation during 1254 and 1935, six $\times$ two lines from each sample of trees were

\begin{tabular}{|c|c|c|c|}
\hline Sample & Number of rings & Start year & End year \\
\hline 1 & 1821 & 64 & 1884 \\
\hline 2 & 1633 & 305 & 1937 \\
\hline 3 & 963 & 1000 & 1962 \\
\hline 4 & 883 & 1053 & 1935 \\
\hline 5 & 818 & 1162 & 1979 \\
\hline 6 & 735 & 1254 & 1988 \\
\hline 7 & 546 & 1443 & 1988 \\
\hline
\end{tabular}

From left to right, they represent the number of samples, total number of annual tree rings, beginning year of tree ring, and end year of tree ring. used. These 12 lines were simply added, and the average value is the variation of the tree rings. We then searched for periodicities in this variation.

\subsection{Analysis results}

Figure 1 shows the annual growth width $d r$ of the Yakusugi, and Figure 2 shows the enlarged graph of the duration during 1200 and 1988 with a 3-year running average curve (black line).

Several dips are apparent. In particular, dips during 1315 to 1370,1425 to 1445,1505 to 1515,1530 to 1600,1645 to 1730,1790 to 1825 , and 1950 to 1980 are apparent. The slow growth rate during 1790 to 1825 corresponds to the Dalton minimum (1795 to 1823). It is notable that three dips during the Spörer minimum coincide with the enhancements of $B e^{10}$ (Beer 2000) and $C^{14}$ (Stuiver 1980). The last dip of 1950 to 1980 may be related to the negative phase of the Pacific Decadal Oscillation (Mantua and Hare 2002; MacDonald and Case 2005).

\subsubsection{Wavelet analysis of tree ring data}

We analyzed the annual variation of the width $d r$ and the area $d S(=2 \pi \mathrm{rdr})$ of the standard tree ring using Fourier and wavelet analysis methods. The result of the wavelet analysis is shown in Figure 3. Quite surprisingly, the wavelet analysis of the annual growth area identified 2 cycles with periodicities of 11 and 24 years during the Oort, Wolf, Spörer, Maunder, and Dalton minima. The 11year periodicity originates from solar activity, while the 24-year periodicity may be induced by the PDO. The vertical axis of Figure 3 corresponds to the inverse of the periodicity (i.e., 0.1 corresponds to a 10 -year periodicity). To see this tendency clearly, the result of the wavelet analysis was applied to a 3-year running average dataset.

\subsubsection{Fourier analyses of tree-ring data}

Figures 4 and 5 represent the result of the Fourier analysis of the datasets of the width $d r$ and the area $d S$ from 1360 to 1988, respectively. Fourier analysis was applied to the data without a running average. A clear peak with an 11-year periodicity and two peaks at 22 and 24 years are identified in both plots. The Fourier analyses of the dataset of $d r$ and $d S$ lead to the same results. It is worth noting that the Fourier analysis also identified a 58-year periodicity and an 89-year periodicity in the variation (Kitagawa and Matsumoto 1995). The existence of 11-year and 22-year periodicities during the Dalton minimum was also confirmed.

\subsubsection{Statistical significance of periodicities}

To investigate the statistical significance of these periodicities, a Monte Carlo (MC) simulation was performed. First, we estimated the fluctuation of the growth rate from the raw data between 1360 and 1988 . 


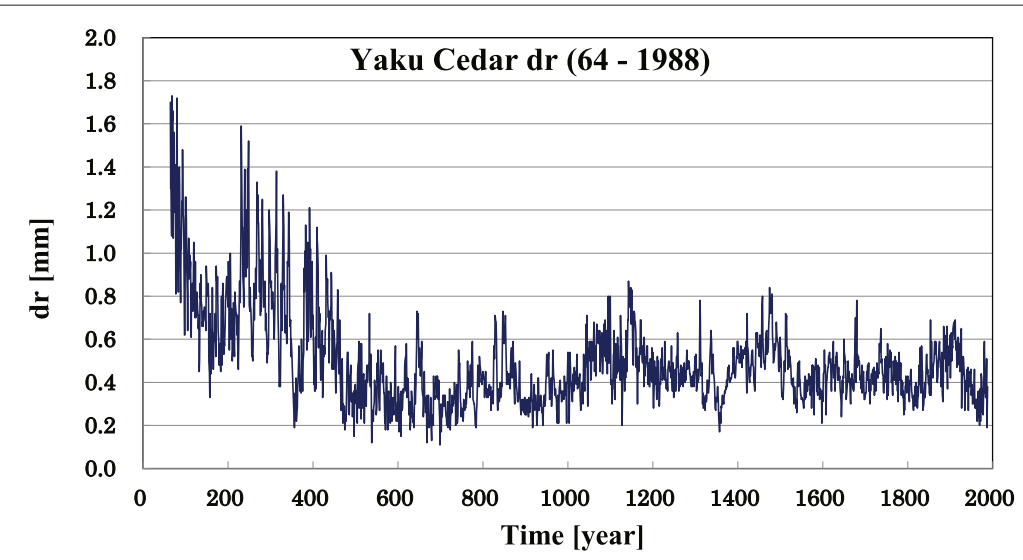

Figure 1 Annual growth width of Yakusugi $d r$ in $\mathbf{m m}$. Numbers on horizontal axis correspond to actual growth years of Yakusugi.

The standard deviation of the yearly growth rate was evaluated to be $0.068 \mathrm{~mm}$ for $d r$ and $400 \mathrm{~mm}^{2}$ for $d S$, with a mean annual growth rate $(<d r\rangle)$ of $0.40 \mathrm{~mm}$ and $(<d S>)$ of $2,250 \mathrm{~mm}^{2}$, respectively. The standard deviation was obtained by fitting the fluctuation to a Gaussian distribution. These fluctuations may be regarded as white noise.

The red noise component was then estimated based on an autoregressive (AR) model. The autoregressive model predicts that the growth rate $d r(t)$ of the year $t$ can be described by the growth rate of 1 year before, $d r(t-1)$, with the relationship $d r(t)=A * d r(t-1)+B$, where $B$ corresponds to random Gaussian noise.

The parameter $A$ can be given by the following equation: $A=\left(\sum_{i=1}^{n}(d r(t)-\right.$ ave. $) *(d r(t-1)-$ ave. $\left.)\right)$ / $\left(\sum_{i=1}^{n}(d r(t)-\text { ave. })^{2}\right)$, where ave. is given by $\sum_{i=1}^{n}$ $(d r(t)) / n$. Based on the data between 1528 and 1885, $A$ is evaluated to be 0.28 for $d r$ and 0.20 for $d S$.
Using these numbers and the above equation, a Monte Carlo calculation was performed to produce a virtual tree ring. The virtual tree ring consists of 629 data points. The number corresponds to the years of the tree ring from 1360 to 1988 . We prepared 20 such samples of virtual tree rings, and a Fourier analysis was applied to the averaged virtual tree ring.

The amplitude spectrum of the Monte Carlo tree ring is given by the red line in Figures 4 and 5, and the amplitude spectrum of the real data is shown by the black line in Figures 4 and 5.

Comparing the red lines at approximately 2.0 for $d r$ and approximately 10,000 for $d S$ with the peak of the 11-year periodicity, we find that the peaks have a statistical significance of $3.1 \sigma$ for $d r$ and $3.0 \sigma$ for $d S$, respectively. The statistical significance for the 22-year periodicity turns out to be $2.7 \sigma$ for $d r$ and $2.5 \sigma$ for $d S$.

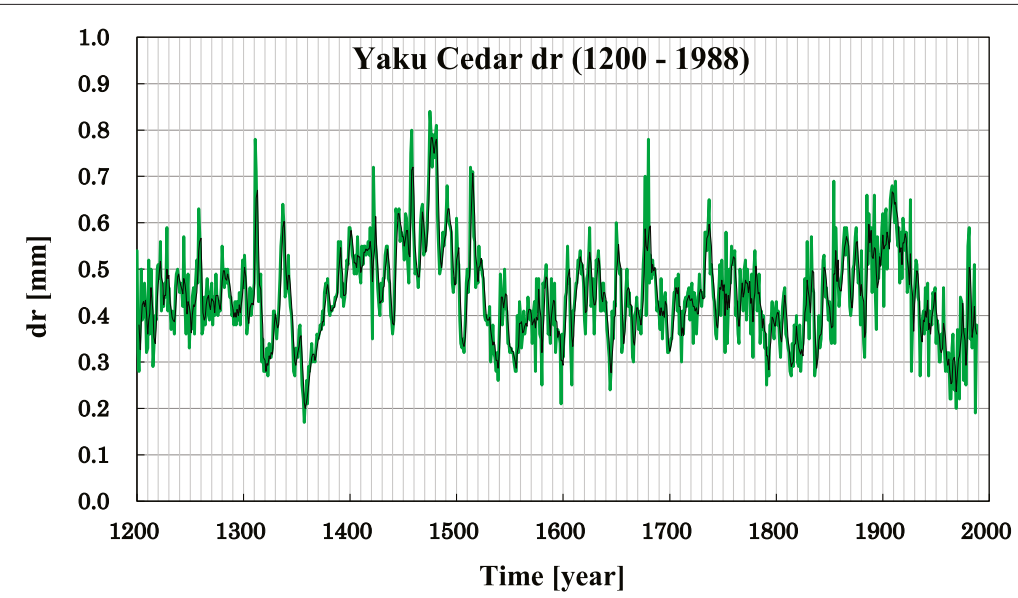

Figure 2 Same data as Figure 1, but for period between 1200 and 1988 with 3-year running average curve (black). Vertical axis represents width of $d r$ in $\mathrm{mm}$. Several dips are apparent in 1315 to 1370,1425 to 1445,1505 to 1515,1530 to 1600,1640 to 1730,1790 to 1825 , and 1950 to 1980. The dip in 1790 to 1825 coincides with the Dalton minimum, 1795 to 1823. 


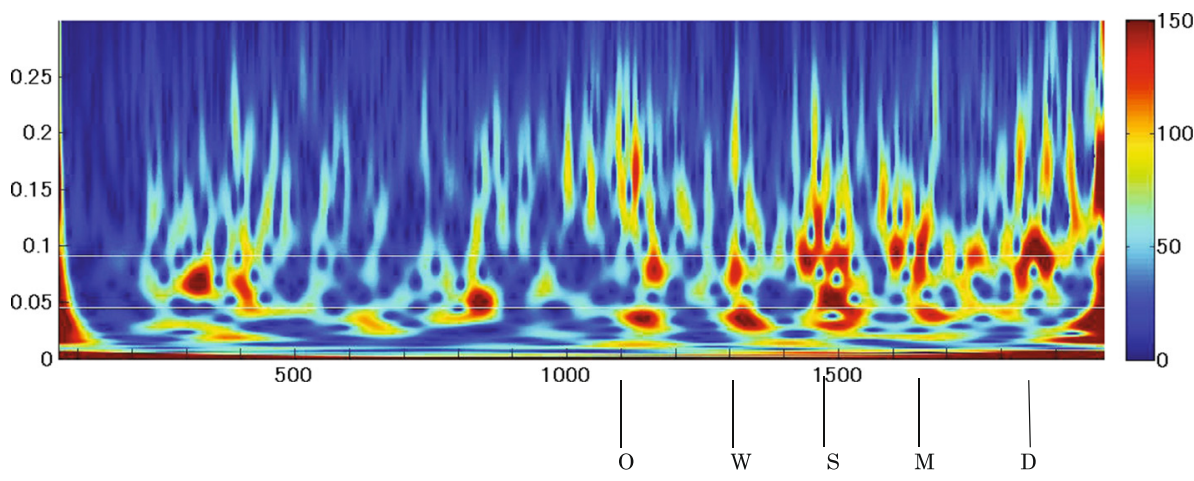

Figure 3 Results of wavelet analysis of annual tree rings of Yakusugi for 3-year average dataset. The 11-year and (24 \pm 4)-year periodicities are apparent between 1100 and 1900. The vertical axis represents the inverse of the periodicity. The periodicities are seen in the pair of 11-year and the (24 \pm 4)-year periodicities and enhanced during the five grand minima. Arrows correspond to each maximum year of $\mathrm{C}^{14}$; from left to right: Oort, Wolf, Spörer, Maunder, and Dalton minima, indicated by arrows in the graph marked O, W, S, M, and D, respectively. The periodicities observed around $320 \mathrm{CE}, 400 \mathrm{CE}, 630 \mathrm{CE}$, and $820 \mathrm{CE}$ may be related to the change in global climate, since each duration coincides with the extension time of Swiss glaciers.

\subsection{Yakushima meteorological data}

In order to understand why periodicities similar to those of the solar cycle exist in the annual tree rings, we examined the meteorological data from Yakushima from 1938 to 2013, with a Fourier analysis. A clear 1-year periodicity was found in the temperature and the water vapor pressure data. A 1-year periodicity of course arises from the four seasons of Yakushima. However, no 11-year periodicity was found in these datasets.

We also analyzed the daylight-hour data. The daylight hour is a quantity defined in meteorology as the hours of a day with sunlight greater than 120 watts $/ \mathrm{m}^{2}$. Looking in

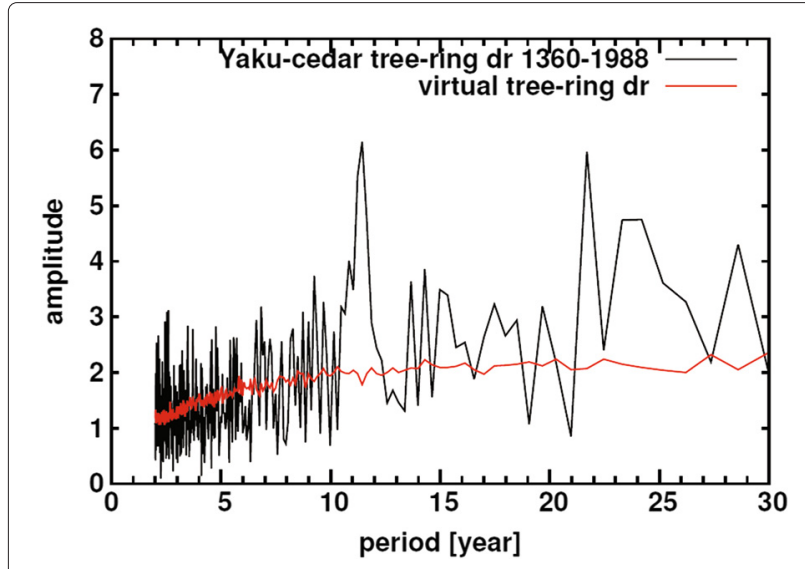

Figure 4 Results of Fourier analysis of $d r$ variation. Analysis was performed for annual tree rings between 1360 and 1988. Dataset without a running average was used. Red line indicates amplitude spectrum for the MC-generated $d r$ data that were randomly produced. Compared to red line, amplitude at 11-year and 22-year periods exceed $3.1 \sigma$ and $2.7 \sigma$, respectively. the solar direction during the daytime with a pyrheliometer and integrating the amount, the daylight hour of a day was determined. When the sunlight was weaker than 120 watts $/ \mathrm{m}^{2}$, we could not identify the shadow on the ground induced by the sun.

Quite surprisingly, not only a 1-year periodicity but also 11-year and (24 \pm 4$)$-year periodicities were found in the daylight-hour data. In particular, the 11-year periodicity is clearly seen in June, while the $(24 \pm 4)$-year periodicity

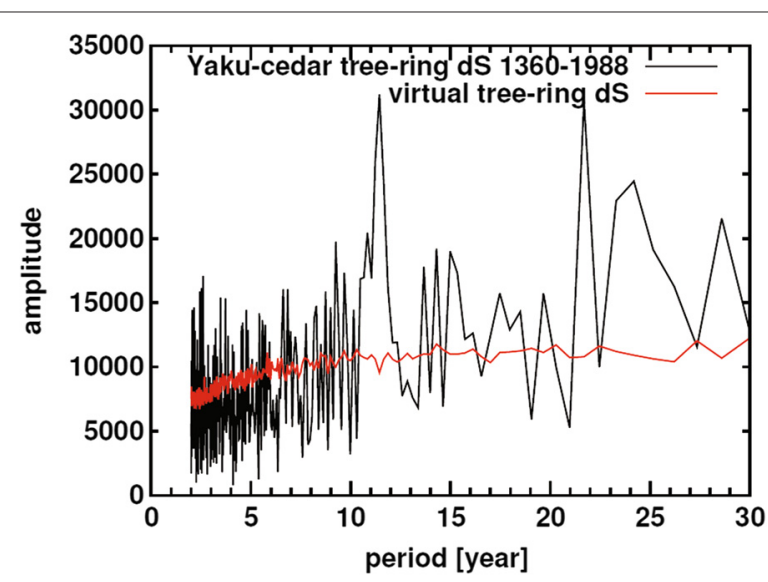

Figure 5 Same results as Figure 4, but for $d S$ variation. Analysis was performed for annual tree rings between 1360 and 1988. Dataset without running average was used. The 11-, 22-, and 24-year periodicities are apparent, confirming the results of the wavelet analysis. Red line indicates amplitude spectrum for MC-generated data that were randomly produced. Compared to the red line, the amplitudes at the 11-year and 22-year periods exceed 3.0 $\sigma$ and $2.5 \sigma$ respectively. Therefore, it would be difficult to explain these peaks as background fluctuation 
may be recognized in the course of a year but appears most clearly in July. The results are shown in Figures 6 and 7 .

It is worthwhile to note that the daylight-hour data shows two clear dips, in 1954 and 1976. These years correspond to periods of minima during the solar cycle during the negative phase of the PDO. We checked the variation of the standard tree ring during these years and confirmed that the Yakusugi grew slowly in times of less sunlight.

\subsubsection{Statistical significance of meteorological data}

In order to estimate the statistical significance of the 11and 22-year periodicities in the daylight-hour spectra of Figures 6 and 7, we used the daylight-hour data of June and July. We estimated the standard deviation as 35.4 hours, with a mean value of 119 hours for June and a mean value of 218 hours with a standard deviation of 50.4 hours for July. The background is produced by the AR model with an autoregression factor of 0.2 . The Monte Carlo simulations were repeated 100 times using these parameters, and the background datasets were created. Applying Fourier analysis to the datasets, the $1 \sigma$ lines were obtained. They are indicated by the red lines in Figures 6 and 7. Comparing these lines with the peaks at 11 years in June and at 22 years in July, the statistical significance of these peaks is estimated to be approximately $2 \sigma$.

\subsection{Summary, discussions, and conclusions}

We analyzed the annual tree rings of the Yakusugi and searched for indication of solar activity. Some very interesting facts were discovered, some of which may have originated from solar activity.

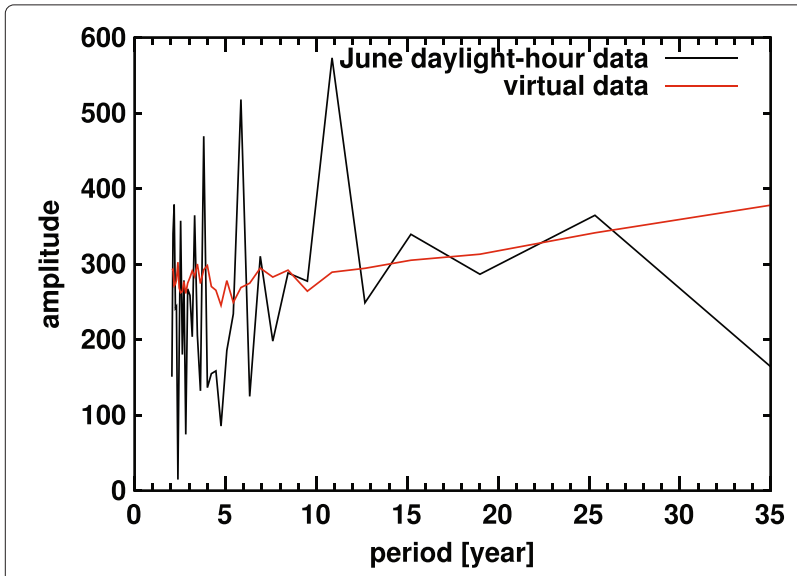

Figure 6 Results of Fourier analysis of daylight-hour data of Yakushima for June of 1938 to 2013 . A clear 11-year periodicity is apparent. Red line indicates amplitude spectrum of background data produced by MC calculation (virtual data). Details of the MC calculations are given in the text.

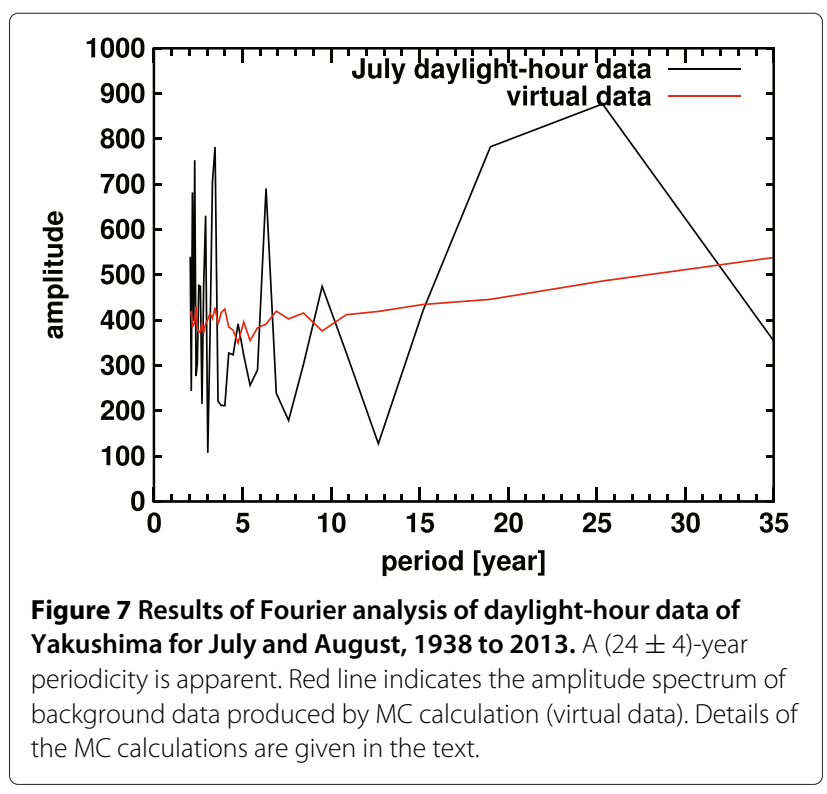

In order to explain the 11-year periodicity, we introduce an assumption: the variation of the growth rate is due to the variation in cloud cover over the island. This hypothesis has been partly checked using the meteorological data of Yakushima from 1938 to 2013.

The daylight-hour data indicates that when solar activity is at a maximum, the daylight hours also increase, while during times of minimum solar activity, the daylight hours decrease.

In general, there are three key factors that affect the photosynthesis of trees: water, temperature, and sunlight. For trees that live on the border of the polar region in Northwest Russia (Kononov et al. 2009) or the pine trees at the highest elevations of western North America (Salzer et al. 2009), temperature is the key factor for growth (the limiting factor), while for pine trees in Arizona, rainfall is the limiting factor (Douglass 1927). However, for the trees in Yakushima, sunlight is the limiting factor; water is not the main factor, since the island experiences rainfall throughout the year. The periodicity recorded in the tree rings of the Yakusugi suggests that during the grand minima, the cloud cover over Yakushima was more influenced by solar irradiance and PDO than it is at present.

The variation in the growth of tree rings due to climate and solar activity was first noticed by Douglass (1927). It is impressive to know that a similar effect is found in a different sample of trees on an isolated island of Japan, on the opposite side of the Pacific Ocean.

Finally, we point out another important result. Figure 3 suggests that pairs of 11-year and (24 \pm 4 )-year periodicities occurred five times, corresponding to the Oort, Wolf, Spörer, Maunder, and Dalton minima. However, we can also identify periodicities around $320 \mathrm{CE}, 400 \mathrm{CE}$, 
$630 \mathrm{CE}$, and $820 \mathrm{CE}$. They may be related to global cold periods, since they coincide with the extension peaks of Swiss glaciers (Holzhauser et al. 2005). Among them, the periodicities around $400 \mathrm{CE}$ and $630 \mathrm{CE}$ may be induced by the solar activity suggested by Usoskin et al. (2007).

\section{Competing interests}

The authors declare that they have no competing interests.

\section{Authors' contributions}

TK measured the tree ring of Yakusugi and prepared the data. SS carried out Fourier analysis for the data and MC calculations. KN carried out the data analysis by the wavelet method. YM analyzed the meteorological data and prepared the manuscript. YM, TM, SS, SK, KM, and KN participated in the discussions and interpretation. All authors read and approved the final manuscript.

\section{Acknowledgements}

The authors acknowledge Prof. Takashi Shibata of the Graduate School of Environmental Studies of Nagoya University for the valuable discussions and Prof. José Valdés-Galicia of UNAM for reading the manuscript. The authors are grateful to Prof. Philip Yock of Auckland University for the useful comments. The authors would also like to acknowledge the Japan Meteorological Agency for providing valuable data from Yakushima for 1938 to 2013.

\section{Author details}

${ }^{1}$ Solar-Terrestrial Environment Laboratory, Nagoya University, 464-8601 Nagoya, Japan. ${ }^{2}$ Nara National Research Institute for Culture Properties, 630-8577 Nara, Japan. ${ }^{3}$ Engineering Science Laboratory, Chubu University, 487-0027 Kasugai, Japan. ${ }^{4}$ Faculty of Science and Technology, Hirosaki University, 036-8577 Hirosaki, Japan

Received: 1 April 2014 Accepted: 27 January 2015

Published online: 27 February 2015

\section{References}

Beer J (2000) Neutron monitor records in broader historical context. Space Sci Rev 93:107-119

Douglass AE (1927) Solar records in tree growth. Science 65(1679):220-221

Holzhauser H, Magny M, Zumbuhl H (2005) Glacier and lake-level variations in west-central Europe over the last 3500 years. The Holocene 15:789-801. 10.1191/0959683605hl853ra

Kitagawa H, Matsumoto E (1995) Climate implication of $\delta C^{13}$ variation in a Japanese cedar during the last two millenia. Geophys Res Lett 22:2155-2158. doi:10.1029/95GL02066

Kononov YM, Friedrich M, Boettger T (2009) Regional summer temperature reconstruction in the Khibiny Low Mountain (Kola Peninsula, NW Russia) by means of tree ring width during the last four centuries. Arctic Antarctic Alpine Res 41:460. doi:10.1657/1938-4246-41.4.460

Lean JL, Rind DH (2008) How natural and anthropogenic influences alter global and regional surface temperatures. Geophys Res Let 35:L18701. 10.1029/2008GL034864

MacDonald GM, Case RA (2005) Variations in the Pacific Decadal Oscillation over the past millennium. Geophys Res Let 32:L08703. doi:10.1029/2005GL022478

Mantua NJ, Hare SR (2002) The Pacific Decadal Oscillation. J Oceanography 58:35-44

Miyahara H, Masuda K, Nagaya K, Kuwana K, Muraki Y, Nakamura N (2004) Cyclicity of solar activity during the Maunder Minimum deduced from radio carbon content. Sol Phys 224:317-322. doi:10.1007/s11207-005-6501-5

Muraki Y, Masuda K, Nagaya K, Wada K, Miyahara H (2011) Solar variability and width of tree ring. Astrophys Space Sci Trans 7:395-401. doi:10.5194/astra-7-395-2011

Rigozo NR, Nordeman DJR, Echer E, Vieira LEA, Echer MPS, Prestes A (2005) Tree-ring width wavelet and spectral analysis of solar variability and climatic effects on a Chilean cypress during the last two and a half millennia. Climate Past Discuss 1:121-135. www.climate-of-the-past.net/cpd/1/121 The related references can be found in the references of this paper. Also the important data of the tree-ring can be obtained from the international tree-ring data bank: http://www.ncdc.noaa.gov/data-access/ paleoclimatology-data/datasets/tree-ring

Salzer MW, Hughes MK (2009) Recent unprecedented tree-ring growth in bristle-cone pine at the highest elevations and possible causes, Kipfmueller KF. PNAS early edition. www.pnas.org/cgi/doi/10.1073/pnas.0903029106

Stuiver M (1980) Atomospheric $C^{14}$ and century-scale solar oscillation. Nature 286:868-871. doi:10.1038/286868a0

Usoskin IG, Solanki SK, Kovaltsov GA (2007) Grand minima and maxima of solar activity: new observational constrains. Astron Astrophys 471:301-309. doi:10.1051/0004-6361:20077704

\section{Submit your manuscript to a SpringerOpen ${ }^{\circ}$ journal and benefit from:}

- Convenient online submission

- Rigorous peer review

- Immediate publication on acceptance

- Open access: articles freely available online

- High visibility within the field

- Retaining the copyright to your article

Submit your next manuscript at $\boldsymbol{\wedge}$ springeropen.com 